\title{
Supplemental table 3.
}

\begin{tabular}{|c|c|c|}
\hline Gene & Forward & Reverse \\
\hline$r p 49$ & AGGGTATCGACAACAGAGTG & CACCAGGAACTTCTTGAATC \\
\hline actin & CCGTACCACAGGTATCGTGTTG & GTCGGTTAAATCGCGACCG \\
\hline cabut & ATGCCTTCTCGCTCTCATGT & TCCTGGAAAGAAGTGGCATC \\
\hline Aldh-III & GATCAAGCCCAGCGAGATT & CGCAGACAACTGGATAGCAA \\
\hline mondo & GCGGCGTTACAACATAAAGA & CTCCATGCGCAAAGCTTCAA \\
\hline$m / x$ & GCCAAGTTTCAAGTGTTCCAG & CTCCAGCCAGGGGATAATG \\
\hline Fas & CTCCACCATCGAGGAGTTCA & CTTGAGCTTGCCAATCCTGT \\
\hline$A C C$ & GGCTATGCTGCGCTTAACA & GCCTCTGTTTTGTGGGTGAC \\
\hline desat1 & GCGCGCCGATCCAATCCTCA & GGTGGCCACAAACCAGGCGT \\
\hline Gs1 & TTGTCCTGTGCGACACCTAC & ATTCTTGCTCAATGCCGAAC \\
\hline Gpdh & GCAAGCTGTCCACCTTCTTC & CAGAAGTCACAAACGCCTCA \\
\hline Gpo-1 & AGATGGGACACTCGGTCAAC & TGATGGAAACGTAGCCCTTC \\
\hline PFK2 & AGAGCGAGTACAACCTGAGC & TAGCGCATTGGCATACTGGT \\
\hline sro & TGCTGGAGTAATGTGCTTCG & TCATGTGTCAGCCTCATGGT \\
\hline
\end{tabular}

